\title{
Branching Ratios, Radiative Lifetimes, and Transition Dipole Moments for Tantalum Nitride, TaN
}

\author{
Jacob L. Bouchard, Timothy Steimle and Damian L. Kokkin \\ School of Molecular Sciences, Arizona State University \\ Tempe, Arizona 85287-1604, U.S.A.
}

David J. Sharfi and Richard J. Mawhorter

Physics and Astronomy Department, Pomona College

Pomona, California 91711, USA

Last Modified: May 19, 2016

*Corresponding Author; Prof. T. C. Steimle, School of Molecular Sciences,

Arizona State University, Tempe, Arizona 85287-1604; Phone:(480)965-3265; eMail: TSteimle@ASU.edu 


\begin{abstract}
The dispersed laser induced fluorescence resulting from excitation in the regions of the $[17.58] 0^{+}-X^{1} \Sigma^{+}(0,0), \quad[18.42] 0^{+}-X^{1} \Sigma^{+}(0,0), \quad[19.22] 1-X^{1} \Sigma^{+}(0,0)$, and $[19.40] 1-X^{1} \Sigma^{+}(0,0)$ bands of tantalum nitride, ${ }^{181} \mathrm{TaN}$, have been recorded and analyzed. The branching ratios and radiative lifetimes for the $[17.58] 0^{+}(v=0),[18.42] 0^{+}(v=0),[19.22] 1^{+}(v=0)$, and $[19.40] 1(v=0)$ states have been determined. From these values the transition dipole moments for visible bands are determined. Vibrational spacing in the $X^{1} \Sigma^{+}$state and the spin-orbit splitting of the $a^{3} \Delta$ state are measured and compared with predicted values. The dispersed fluorescence spectra and determined branching ratios reveal that the most effective mechanism for populating the $a^{3} \Delta_{1}(J=1, v=0)$ state, which will be used in future $P$ - and $T$-violation measurements, is via excitation of the [18.42]0 $0^{+-} X^{1} \Sigma^{+}(0,0)$ band followed by subsequent spontaneous or stimulated emission.
\end{abstract}




\section{INTRODUCTION}

Searches for permanent electric dipole moments, EDMs, of nucleons, nuclei, atoms, and molecules are effective, low-energy, venues for testing the Standard Model (SM) ${ }^{1-3}$. To date no EDM, which are $P$ - and $T$-violating, has been measured. Permanent EDMs of elementary particles are highly suppressed in the SM (electron: $\left|d_{e}^{S M}\right|<10^{-38} e \cdot \mathrm{cm}$; proton and neutron: $\left|d_{n, p}^{S M}\right|<10^{-32} e \cdot \mathrm{cm} \cdot 10^{-38} e \cdot \mathrm{cm}$ ), whereas newer theories often predict orders of magnitude larger values. Paramagnetic diatomic molecules have for some time ${ }^{4}$ been in the spotlight as venues for permanent EDM searches. The EDM of atoms and molecules arises from both the sum of the intrinsic EDM of the constituent particles and the mixing of opposite parity states due to $T, P$ odd interactions. The EDM of paramagnetic molecules is dominated by the former ${ }^{2}$. Along these lines, significant efforts have been made to establish increasingly lower upper limits on the electron's electric dipole moment (eEDM), $\left|\mathrm{d}_{e}\right|$, which is currently $8.7 \times 10^{-29} e \cdot \mathrm{cm}$ and was obtained using the $H^{3} \Delta_{1}(v=0, J=1)$ state of thorium oxide, $\mathrm{ThO}^{5}$. A variety of other heavy paramagnetic diatomic molecules are also being used in attempts to measure $\left|\mathrm{d}_{e}\right|^{6}$.

In the hadron sector, the $T, P$-odd magnetic quadrupole moment (MQM) of the nucleus is another source of an atomic or molecular $\mathrm{EDM}^{2,4,7-9}$. Recently it was found that the sensitivity to this $T, P$-odd effect could be high in paramagnetic molecules containing deformed nuclei ${ }^{8,9}$. In this case the EDM is generated via the interaction of the nuclear MQM with the gradient of the magnetic field produced by the electrons. This interaction mixes states of opposite parity resulting in an EDM. The nuclear MQM induced EDM is postulated to be 1-2 orders of magnitude larger than that induced by the Schiff moment ${ }^{3,8}$. It is believed that if measurements of EDM-induced frequency shifts can be made with sensitivity of only an order of magnitude better than what has been achieved in the recent eEDM experiment ${ }^{5}$, then limits on several underlying parameters of hadronic $T, P$-violation can be improved ${ }^{8}$. Heavy diatomic molecules in ${ }^{3} \Delta_{1}$ states are particularly promising venues for investigations of nuclear-MQM induced EDMs for many of the same reasons that such molecules and states are attractive for eEDM measurements. Both ${ }^{229} \mathrm{ThO}$ and ${ }^{181} \mathrm{TaN}$ in their low-lying metastable ${ }^{3} \Delta_{1}$ states are candidates ${ }^{8}$ 
for nuclear-MQM induced EDMs measurements. The $a^{3} \Delta_{1}$ state ${ }^{181} \mathrm{TaN}$ is now also being considered as venue for eEDM measurements.

Tantalum nitride, ${ }^{181} \mathrm{TaN}$, the subject of the present report, is particularly attractive for nuclear MQM induced EDM studies in part because there is one dominant stable isotope, ${ }^{181} \mathrm{Ta}$ (99.99\%), and because there is a strong enhancement of its nuclear MQM due to the collective nature $^{2,10}$ of this deformed nucleus. Collectivity is also the cause of the large nuclear electric quadrupole moment $\left(=+386 \mathrm{fm}^{2}\right)$ of ${ }^{181} \mathrm{Ta}$. The lower excitation energy of the $a^{3} \Delta_{1}$ state ${ }^{181} \mathrm{TaN}$, as compared to the $H^{3} \Delta_{1}$ state ${ }^{229} \mathrm{ThO}$, may enhance the lifetime and hence improve the sensitivity $^{8}$ of both nuclear MQM induced EDMs and eEDM measurements. In support of the propose EDM measurements, Skripnikov, et al. ${ }^{11}$ predicted the nuclear-MQM electronicmagnetic-field interaction constant, $W_{M}$, the eEDM interaction constant, $W_{d}$, the electron-nucleon scalar-pseudoscalar interaction constant, $W_{P, T}$, molecule-frame electric dipole moment, $\vec{\mu}_{\mathrm{el}}$, the magnetic hyperfine structure constant, $A_{\|}$, equilibrium internuclear separation, $R_{e}$, and vibrational parameters, $\omega_{e}$ and $\omega_{e} x_{e}$,for the $a^{3} \Delta_{1}$ state. A two-step procedure to investigate the four-component electronic structure in the vicinity of the Ta nucleus was performed. More recently, the properties of $\mathrm{TaN}$ were predicted by Fleig, et $a .^{12}$ using an all-electron, configuration interaction, method using the Dirac-Coulomb Hamiltonian. In addition to predicting $W_{M}, W_{d}, W_{P, T}, A_{\|}, \vec{\mu}_{\mathrm{el}}, R_{e}$ and $\omega_{e}$ for the $a^{3} \Delta_{1}$ state, the $\vec{\mu}_{\mathrm{el}}, R_{e}$, and $\omega_{e}$ constants for the $X^{1} \Sigma^{+}$state and the $X^{1} \Sigma^{+} \rightarrow a^{3} \Delta_{1}$ electric dipole transition moment were predicted. The predicted values were in good agreement with those of Skripnikov, et al. ${ }^{11}$.

There is relatively little experimental information for TaN. The visible absorption spectrum of an argon matrix isolated sample was first identified in $1979^{13}$ and the strong bands assigned to progressions in singlet-to-singlet electronic transitions. The infrared absorption spectrum of argon matrix isolated TaN has also been recorded ${ }^{14}$. More recently, Ram et al. ${ }^{15}$ have recorded and analyzed the emission spectra for high-temperature, gas-phase, TaN in the $3000-35,000 \mathrm{~cm}^{-1}$ range using Fourier transform spectroscopy. Twenty one bands in the emission spectrum were assigned and rotationally analyzed. The precision of the strong lines were approximately \pm 0.003 $\mathrm{cm}^{-1}$ and those of the weaker lines $\pm 0.005 \mathrm{~cm}^{-1}$. The lowest branch features were not measured due to the high temperature. The electronic energy $a^{3} \Delta_{1}$ was determined to be $2827.2917(24) \mathrm{cm}^{-}$ 
1. The assignment of the spectrum was aided by a large scale CASSCF/CMRCI ab initio calculation of the valence electronic states having energies less than $25,000 \mathrm{~cm}^{-1}$. Quasirelativistic pseudopotentials were used to describe the core electrons. Spin-orbit coupling effects were not included. Fourteen Hund's case (a) electronic states, corresponding to seven singlet, five triplet, and two quintet states are predicted in this range. The $X^{1} \Sigma^{+}$state was predicted to have a dominant $1 \sigma^{2} 2 \sigma^{2} 1 \pi^{4} 3 \sigma^{2}$ configuration. The $a^{3} \Delta$ state was predicted to lie $4276 \mathrm{~cm}^{-1}$ above the $X^{1} \Sigma^{+}$state and have a dominant $1 \sigma^{2} 2 \sigma^{2} 1 \pi^{4} 3 \sigma^{1} 1 \delta^{1}$ configuration. In these configurations the $1 \sigma, 2 \sigma$, and $1 \pi$ orbitals are bonding orbitals formed between the $\mathrm{N}(2 p)$ orbital and the $\operatorname{Ta}(5 p / 5 d)$ hybrid orbitals, whereas the $1 \delta$ is essentially a pure $\operatorname{Ta}\left(5 d_{ \pm 2}\right)$ orbital and the $3 \sigma$ orbital is a $5 d_{0} / 6 s$ hybrid orbital polarized away from the electrophilic $\mathrm{N}$-center.

Here we report on the first spectroscopic study of supersonically cooled TaN sample. Laser excitation and subsequent dispersed fluorescence of four band systems in the green and yellow spectral range were investigated. The excitation bands are the strong transitions previously observed in emission ${ }^{15}:[17.58] 0^{+}-X^{1} \Sigma^{+}(0,0), \quad[18.42] 0^{+}-X^{1} \Sigma^{+}(0,0), \quad[19.22] 1-$ $X^{1} \Sigma^{+}(0,0)$, and [19.40]1- $X^{1} \Sigma^{+}(0,0)$. Here a $\left[T_{\mathrm{v}} / 1000\right]|\Omega|$ designation is used for the excited state because a Hund's case (a) electronic states designation of ${ }^{2 S+1}|\Lambda|_{\Omega}$ is not appropriate due to the expected large spin-orbit interaction. Although the $a^{3} \Delta_{1}(J=1, v=0)$ level will be used for investigations of the nuclear MQM induced EDMs and eEDM measurements, the strong visible bands of the present work involving the $X^{1} \Sigma^{+}(v=0)$ state are relevant for several reasons. Similar to the scheme for the ThO eEDM measurements ${ }^{5}$, these transitions offer a route for populating the requisite $a^{3} \Delta_{1}(J=1, v=0)$ level via optical pumping the $X^{1} \Sigma^{+}(J=0$ and 1$) \rightarrow$ $[17.58] 0^{+}(J=0$ and 1$),[18.42] 0^{+}(J=0$ and 1$),[19.22] 1(J=1)$, and $[19.40] 1(J=1)$ transition which spontaneous emit to the $a^{3} \Delta_{1}(J=1, v=0)$ level. For detection in the proposed measurements, a transition from the $a^{3} \Delta_{1}(J=1, v=0)$ state to another higher energy $|\Omega|=1$ state that strongly fluoresces to the $X^{1} \Sigma^{+}$state in the green, blue, or near ultraviolet spectral range, where there are highly efficient detectors, is desirable. It is also desirable that fluorescence be predominantly at one wavelength (i.e. branching ratio of approximately 1 to the $X^{1} \Sigma^{+}(v=0)$ state). Laser-based spectroscopic studies of these bands may also be relevant to future efforts at 
trapping and cooling TaN. Optical forces can be used to transversely cool and collimate beams of TaN so as to enable higher downstream flux and hence improved statistical sensitivity. Efficient laser cooling requires that the molecules have an intense electronic transition and fluoresce to a minimal number of rovibronic levels ${ }^{16}$. The nuclear electric quadrupole splitting, of each rotational level, which is currently unknown, could make laser cooling impractical. Finally, the experimental determination of the nuclear MQM induced EDMs and eEDM require accurate predictions of $W_{M}, W_{d}$, and $W_{P, T}$. The quality of the relativistic electronic structure calculations used to predict these interaction constants can be most readily assessed by comparing experimentally determined properties such as energies, magnetic and nuclear electric quadrupole hyperfine parameters, bond lengths, molecular frame dipole moments, and vibrational parameters, with predicted values.

\section{EXPERIMENTAL}

The production scheme used in the present study is similar to that used to characterize $\mathrm{ThO}^{17}$. A continuously rotating tantalum metal rod was ablated in a supersonic expansion of $3 \%$ $\mathrm{NH}_{3}$ seeded in an argon carrier gas with a backing pressure of approximately $3 \mathrm{MPa}$. The supersonic expansion was then crossed with pulsed laser radiation approximately $10 \mathrm{~cm}$ downstream from the source. The excitation laser was a $20 \mathrm{~Hz}$, excimer pumped, pulsed dye laser having a spectral resolution of approximately $0.1 \mathrm{~cm}^{-1}$. A commercial wavemeter was used to determine the absolute wavenumber of the laser. Three types of experiments were preformed: (a) laser induced fluorescence (LIF) excitation spectroscopy in the ranges covering the $[17.58] 0^{+}-X^{1} \Sigma^{+}(0,0), \quad[18.42] 0^{+}-X^{1} \Sigma^{+}(0,0), \quad[19.22] 1-X^{1} \Sigma^{+}(0,0)$, and $[19.40] 1-X^{1} \Sigma^{+}(0,0)$ bands, (b) dispersed fluorescence (DF) emission spectroscopy in the 500-750 nm range, and (c) fluorescence lifetime measurements. The excitation spectra were extracted from two dimensional (2D) spectra as described previously ${ }^{17}$. Briefly, a 2D spectra has as one of the dimensions the laser excitation wavelength and as the other dimension the wavelength of the DF. In the set up used here a $75 \mathrm{~nm}$ wide spectral window in the DF is simultaneously monitored using a cooled, gated, intensified charge coupled device (CCD) detector attached to a $2 / 3 \mathrm{~m}$ monochromator. The entrance slit width of the monochromator was set to approximately $0.75 \mathrm{~mm}$ resulting in a spectral resolution of approximately $\pm 2 \mathrm{~nm}$ for the DF. The gate for the CCD detector was typically $1 \mu$ s wide with a 20 ns delayed from the laser pulse to reduce scattered laser light. The 
central wavelength of the $75 \mathrm{~nm}$ wide spectral window was usually selected to coincide, and be tracked, with the wavelength of the laser (i.e. the central wavelength detect the on-resonance emission). Typically in recording the 2D spectra, DF signals from 50 free-jet expansion pulses were averaged at a given laser excitation wavelength. The laser excitation was then stepped by $0.1 \mathrm{~cm}^{-1}$ and the process repeated. The excitation spectra presented here were extracted from the 2D spectra by summing the appropriate wavelength regions of the DF.

The DF spectra were obtained by fixing the excitation wavelength of the pulsed laser at that corresponding to either $Q$-branch or $R$-branch head and recording $75 \mathrm{~nm}$ wavelength segments of the resulting emission. In these measurements the entrance slits on the monochromator were narrowed to approximately $0.25 \mathrm{~mm}$ resulting in a spectral resolution of approximately $\pm 0.7 \mathrm{~nm}$. Typically in this mode of operation 10000 averages were taken for each $75 \mathrm{~nm}$ monochromator transmission window. The DF signal at a given emission wavelength was obtained from vertical binning of the 2D CCD detector. The multiple 75-nm-wide, one-dimensional arrays of intensity versus dispersed fluorescence wavelength were spliced together, and adjusted for wavelength variation of sensitivity, to produce the DF spectra. Wavelength calibration of the DF spectra was achieved by recording the emission of an argon pen lamp. The calibration lines also facilitated the splicing together of the 75-nm-wide segments. A gate delay of 75 ns was used in the DF measurements to eliminate laser scatter contributions to the on-resonance emission signal and hence possible distortion to the determined branching ratios.

The fluorescence decay curves were measured by tuning the laser wavelength to be resonant with a particular feature of the excitation spectra and recording the DF spectrum at variable time delays from the pulsed laser. Typically, the gate for the CCD detector was set to a large value (approximately $2 \mu \mathrm{s}$ ) and the time delay from the laser excitation stepped in 20-ns increments to produce a series of DF spectra. The resulting DF spectra were integrated over the appropriate wavelength region to give the relative fluorescence intensity at a given delay from the laser excitation.

\section{OBSERVATIONS}

A diagram of the relevant energy levels associated with the detected electronic transitions can be found in Ref. 15. Laser excitation spectra in the region of the $[17.58] 0^{+}-X^{1} \Sigma^{+}(0,0)$, 
$[18.42] 0^{+}-X^{1} \Sigma^{+}(0,0), \quad[19.22] 1-X^{1} \Sigma^{+}(0,0)$, and $[19.40] 1-X^{1} \Sigma^{+}(0,0)$ bands extracted from 2D spectra are presented in Figure 1. The $[17.58] 0^{+}-X^{1} \Sigma^{+}(0,0)$ and $[18.42] 0^{+}-X^{1} \Sigma^{+}(0,0)$ bands exhibit only $P$ and $R$ branches expected for a $\Delta \Omega=0$ transition, while the [19.22]1- $X^{1} \Sigma^{+}$ $(0,0)$ and [19.40]1- $X^{1} \Sigma^{+}(0,0)$ bands exhibit $P, Q$, and $R$ branches as expected for an $\Delta \Omega=1$ transition. Also evident in the excitation spectrum is a band near $18408 \mathrm{~cm}^{-1}$ which has been previously seen in emission ${ }^{15}$ and assigned as the $0^{+} \rightarrow X^{1} \Sigma^{+}(v=1)$ transition. Using the energy for the $X^{1} \Sigma^{+}(v=1)$ state derived from the DF spectra (infra vide) of $1070 \pm 4 \mathrm{~cm}^{-1}$, the energy of the $0^{+}$state is $19478 \pm 4 \mathrm{~cm}^{-1}$ and this band can now be designated as the $[19.48] 0^{+}-X^{1} \Sigma^{+}(0,1)$ transition. The spectral feature at $19387 \mathrm{~cm}^{-1}$ is the $a^{4} \mathrm{~F}_{9 / 2} \rightarrow z^{6} \mathrm{~F}_{11 / 2}$ transition of atomic Ta. Note that the $R$-branch head occurs at a higher rotational quantum number for the $[17.58] 0^{+}-$ $X^{1} \Sigma^{+}(0,0)$ band than in the other bands, which is consistent with the previously determined ${ }^{15}$ ordering of rotational constants, $B$. The observed relative intensities of the well resolved $P$ branches lines of the $[17.58] 0^{+}-X^{1} \Sigma^{+}(0,0)$ and $[18.42] 0^{+}-X^{1} \Sigma^{+}(0,0)$ bands gives a rotation temperature of approximately $15 \mathrm{~K}$.

The DF spectra, and associated assignment, resulting from excitation near the $R$-branch head of the $[17.58] 0^{+}-X^{1} \Sigma^{+}(0,0)$ band $\left(v=17591 \mathrm{~cm}^{-1}\right)$ and the $R$-branch head of the [18.42] $0^{+-}$ $X^{1} \Sigma^{+}(0,0)$ band $\left(v=18429 \mathrm{~cm}^{-1}\right)$ are presented in Figure 2 . The decreasing quantum efficiency of the CCD detector causes the signal to noise ratio $(\mathrm{S} / \mathrm{N})$ to decrease in the red. Emission resulting from excitation of the $[17.58] 0^{+}-X^{1} \Sigma^{+}(0,0)$ band is very diagonal whereas that resulting from excitation of the $[18.42] 0^{+}-X^{1} \Sigma^{+}(0,0)$ band exhibits a $X^{1} \Sigma^{+}(\nu=0,1,2)$ vibrational progression as well as emission to the $a^{3} \Delta_{1}(v=0,1)$ levels. The DF spectra, and associated assignment, resulting from excitations near the $R$-branch head $\left(v=19225 \mathrm{~cm}^{-1}\right)$ and $Q$-branch head $\left(v=19217 \mathrm{~cm}^{-1}\right)$ of the $[19.22] 1-X^{1} \Sigma^{+}(0,0)$ band are presented in Figure 3. The $R$-branch and $Q$-branch excitations populate different parity levels of the [19.22]1 state. The DF spectra resulting from the $e$-parity level, which are populated via $R$-branch head excitation, is somewhat weaker than that resulting from the $f$-parity levels, which are populated via $Q$-branch head excitation. This difference in intensity is primarily because the $Q$-branch head is more intense than the $R$-branch head (Figure 1) and not due to an intrinsic difference of the transition dipoles for the emission for the two parity components. The DF spectra, and associated assignment, 
resulting from excitations near the $R$-branch head $\left(v=19403 \mathrm{~cm}^{-1}\right)$ and $Q$-branch head $(v=19396$ $\left.\mathrm{cm}^{-1}\right)$ of the [19.40]1- $X^{1} \Sigma^{+}(0,0)$ band are presented in Figure 4. In this case the DF spectrum resulting from the $e$-parity levels is of comparable intensity to the spectrum resulting from the $f$ parity levels because the $R$-branch and $Q$-branch heads are of comparable intensity in the excitation spectrum (Figure 1). The weak features near $680 \mathrm{~nm}$ and $740 \mathrm{~nm}$ are not readily assigned and have been omitted in the determination of the branching ratios. Although the wavelengths of these features are near those associated with the $[17.36] 2(v=0) \rightarrow$ $a^{3} \Delta_{1}(v=0$ and 1$)$ emission, a sequential $[19.40] 1 \rightarrow[17.36] 2(v=0) \rightarrow a^{3} \Delta_{1}(v=0$ and 1$)$ fluorescence process seems unlikely because the first step would be very improbable given the low frequency. An alternative assignment is [19.40]1 $\rightarrow a^{3} \Delta_{1}(v=2$ and 3), but it is difficult to rationalize given the absence of $[19.40] 1 \rightarrow a^{3} \Delta_{1}(v=0$ and 1$)$ features. The assignment and measured relative energies of the lower state for the DF spectra resulting from the $[17.58] 0^{+}(v=0), \quad[18.42] 0^{+}(v=0),[19.22] 1(v=0)$, and [19.40]1 $(v=0)$ vibronic levels are given in Tables I. The error associated with the relative lower state energies is estimated to be $\pm 4 \mathrm{~cm}^{-1}$.

Observed and calculated radiative decay curves resulting from the laser excitation near the $R$-branch heads of the $[17.58] 0^{+}-X^{1} \Sigma^{+}(0,0)$ and $[18.42] 0^{+}-X^{1} \Sigma^{+}(0,0)$ bands and the $Q$-branch heads [19.22] $1-X^{1} \Sigma^{+}(0,0)$ and $[19.40] 1-X^{1} \Sigma^{+}(0,0)$ bands are presented in Figure 5. In each case resonant fluorescence was monitored. There was no noticeable difference in the decay curves for the $R$-branch fluorescence with those obtained from $Q$-branch fluorescence for the $[19.22] 1-X^{1} \Sigma^{+}(0,0)$ and $[19.40] 1-X^{1} \Sigma^{+}(0,0)$ bands.

\section{ANALYSIS AND DISCUSSION}

Analysis of the DF data places the $a^{3} \Delta_{1}(v=0)$ state at $2833 \pm 4 \mathrm{~cm}^{-1}$ above the $X^{1} \Sigma^{+}(v=0)$ state, which compares well with the previous ${ }^{15}$, more precisely determined, value of $2827.2917(24) \mathrm{cm}^{-1}$. Similarly, the $a^{3} \Delta_{2}(v=0)$ state is determined to be located $4023 \pm 4 \mathrm{~cm}^{-1}$ above the $X^{1} \Sigma^{+}(v=0)$ state giving an observed spin-orbit splitting of $1195 \pm 4 \mathrm{~cm}^{-1}$. The most recent electronic structure calculation ${ }^{12}$ performed at identical level of theory predicts the energies of the $a^{3} \Delta_{1}(v=0)$ and $a^{3} \Delta_{2}(v=0)$ levels to be $2526 \mathrm{~cm}^{-1}$ and $3618 \mathrm{~cm}^{-1}$, 
respectively, giving a predicted spin-orbit splitting to be $1092 \mathrm{~cm}^{-1}$. The analysis of the DF data also gives a vibrational spacing between the $v=0$ and $v=1$ levels of the $X^{1} \Sigma^{+}$state to be $1070 \pm 4 \mathrm{~cm}^{-1}$, whereas the predicted values are $1049 \mathrm{~cm}^{-1}$ (Ref. 15) and $1134 \mathrm{~cm}^{-1}$ (Ref. 12). The value from the analysis of the matrix isolated infrared spectrum ${ }^{14}$ is also $1070 \mathrm{~cm}^{-1}$.

The integrated intensities of the DF spectral features of Figures 2, 3, and 4 were used to estimate the branching ratios, in percentage (\%), given in Tables I. The CCD detector is insensitive at wavelengths greater than about $750 \mathrm{~nm}$ and the determined branching ratios assume that there is no emission to the red of $750 \mathrm{~nm}$. The assumption of ignoring the near infrared emission is partly justified by the $v^{3}$ dependence of the spontaneous emission. The DF spectra and determined branching ratios reveal that the most effective mechanism for populating the $a^{3} \Delta_{1}(J=1, v=0)$ state, which will be used in an attempt to measure the nuclear MQM induced EDMs and eEDM, is via excitation of the $[18.42] 0^{+}-X^{1} \Sigma^{+}(0,0)$ band followed by subsequent spontaneous or stimulated emission. The branching ratio for the $[18.42] 0^{+} \rightarrow$ $a^{3} \Delta_{1}(v=0)$ emission is $52.9 \%$. Also given in Table I are estimates for the upper limit for the branching ratios and associated transition dipole moment for features not detected in the DF spectra. These estimates were obtained by considering the observed $\mathrm{S} / \mathrm{N}$ at the expected wavelength and $v^{3}$ dependence.

The fluorescence decay curves of Figure 5 were fit to a single exponential function to give the radiative lifetimes of $454 \pm 32 \mathrm{~ns}, 479 \pm 12 \mathrm{~ns}, 333 \pm 4 \mathrm{~ns}$, and $480 \pm 17 \mathrm{~ns}$, respectively, for the $[17.58] 0^{+},[18.42] 0^{+},[19.22] 1$, and $[19.40] 1$ states. The similarity of the values for the $[17.58] 0^{+},[18.42] 0^{+}$, and $[19.40] 1$ states suggest that they arise from the same primary electronic configuration whereas that for the [19.22]1 state is different. Following the same procedure as in ThO study ${ }^{17}$, the radiative lifetimes were combined with the branching ratios to determine the transition dipole moments. The values associated with the $[17.58] 0^{+},[18.42] 0^{+},[19.22] 1$, and [19.40]1 states are given in Table I. The radiative lifetime of $454 \mathrm{~ns}$ and the approximate unity branching ratio for the $[17.58] 0^{+}-X^{1} \Sigma^{+}(0,0)$ band gives a transition dipole of $1.134 \mathrm{D}$. 


\section{CONCLUSION}

An efficient method for the production and detection of a cold molecule beam sample of ${ }^{181} \mathrm{TaN}$ has been demonstrated. The determined branching ratios, radiative lifetimes, and transition dipole moments should facilitate design of experiments to measure the nuclear MQM induced EDM and eEDM. An effective experimental scheme for populating the $a^{3} \Delta_{1}(J=1, v=0)$ state has been identified. The determined spin-orbit splitting between the $a^{3} \Delta_{1}(v=0)$ and $a^{3} \Delta_{2}(v=0)$ levels of $1195 \pm 4 \mathrm{~cm}^{-1}$ is in fair agreement with the predicted value $^{12}$ of $1092 \mathrm{~cm}^{-1}$. High resolution laser excitation spectroscopic studies of the [17.58] $0^{+-}$ $X^{1} \Sigma^{+}(0,0), \quad[18.42] 0^{+}-X^{1} \Sigma^{+}(0,0), \quad[19.22] 1-X^{1} \Sigma^{+}(0,0)$, and $[19.40] 1-X^{1} \Sigma^{+}(0,0)$ bands

presented here, as well as those involving excitation from the $a^{3} \Delta_{1}(v=0)$ state, are currently in progress.

\section{Acknowledgements}

This research has been supported by the National Science Foundation, Division of Chemistry, CHE-1265885 (ASU) and the Pomona College Sontag Fellowship Program. 
Table I. The branching ratio (BR) and transition dipole moments (TD).

\begin{tabular}{|c|c|c|c|c|c|c|c|c|c|c|c|c|c|c|}
\hline \multirow{2}{*}{ Band $^{\mathrm{a}}$} & \multirow[t]{2}{*}{ Assignment $^{\mathrm{b}}$} & \multirow[t]{2}{*}{ Energy $^{c}$} & $\mathrm{BR}(\%)$ & TD & $\mathrm{BR}(\%)$ & $\mathrm{TD}^{\mathrm{c}}$ & $\mathrm{BR}(\%)$ & TD & $\mathrm{BR}(\%)$ & $\mathrm{TD}$ & $\mathrm{BR}(\%)$ & TD & $\mathrm{BR}(\%)$ & $\mathrm{TD}$ \\
\hline & & & \multicolumn{2}{|c|}{$[17.58] 0^{+}$} & \multicolumn{2}{|c|}{$[18.42] 0^{+}$} & \multicolumn{2}{|c|}{$[19.22] 1-Q$} & \multicolumn{2}{|c|}{ [19.22]1-R } & \multicolumn{2}{|c|}{$[19.40] 1-Q$} & \multicolumn{2}{|c|}{$[19.40] 1-R$} \\
\hline A & $X^{1} \Sigma^{+}(\nu=0)$ & 0.000 & 100 & 1.13 & 21.0 & 0.49 & 39.6 & 0.73 & 49.9 & 0.82 & 65.2 & 0.82 & 73.8 & 0.86 \\
\hline B & $X^{1} \Sigma^{+}(\nu=1)$ & 1073 & $(2)^{\mathrm{d}}$ & $\left(0.2^{\mathrm{d}}\right.$ & 16.0 & 0.46 & 1.2 & 0.14 & $(1)$ & $(0.1)$ & $(1)$ & $(0.1)$ & 2.2 & 0.16 \\
\hline $\mathrm{C}$ & $X^{1} \Sigma^{+}(v=2)$ & 2123 & (3) & $(0.2)$ & 2.6 & 0.21 & (1) & $(0.1)$ & (2) & $(0.2)$ & (1) & $(0.1)$ & (1) & $(0.1)$ \\
\hline $\mathrm{D}$ & $a^{3} \Delta_{1}(v=0)$ & 2833 & (5) & $(0.3)$ & 52.9 & 0.99 & 2.1 & 0.22 & (3) & $(0.2)$ & (1) & $(0.1)$ & (2) & $(0.2)$ \\
\hline $\mathrm{E}$ & $a^{3} \Delta_{1}(v=1)$ & 3837 & (5) & $(0.3)$ & 7.5 & 0.41 & 1.6 & 0.21 & (4) & $(0.3)$ & (2) & $(0.2)$ & (2) & $(0.2)$ \\
\hline $\mathrm{G}$ & $a^{3} \Delta_{2}(v=1)$ & 5070 & - & - & (3) & $(0.3)$ & 2.6 & 0.30 & 4.9 & 0.41 & 3.6 & 0.29 & 3.9 & 0.31 \\
\hline
\end{tabular}

a) The spectral feature identified in Figures 2-4.

b) The assignment of the lower energy terminus of the DF spectral feature.

c) Relative energy of lower state obtained from measured wavelengths of DF spectra. Estimated error $\pm 4 \mathrm{~cm}^{-1}$.

d) Transition dipole in Debye.

e) The numbers in parentheses are estimated upper limits for BR and TD for those transitions not detected. 


\section{Figure Captions}

\section{Figure 1.}

Broad laser excitation spectra in the region of the: a) $[19.40] 1-X^{1} \Sigma^{+}(0,0)$, b) $[19.22] 1-X^{1} \Sigma^{+}$ $(0,0), \mathrm{c})[18.42] 0^{+}-X^{1} \Sigma^{+}(0,0)$, and d) $[17.58] 0^{+}-X^{1} \Sigma^{+}(0,0)$, bands. The $[19.45] 0^{+}-X^{1} \Sigma^{+}(0,1)$ band near $18408 \mathrm{~cm}^{-1}$ also indicated. The spectral feature at $19387 \mathrm{~cm}^{-1}$ is the $a^{4} \mathrm{~F}_{9 / 2} \rightarrow z^{6} \mathrm{~F}_{11 / 2}$ transition of atomic Ta.

\section{Figure 2.}

The DF spectra resulting from excitations near the $R$-branch head of the: a) $[17.58] 0^{+}-X^{1} \Sigma^{+}$ $(0,0)$ band, b) $[18.42] 0^{+}-X^{1} \Sigma^{+}(0,0)$ band. Also show is the associated vibronic levels.

\section{Figure 3.}

The DF spectra resulting from excitations near the $R$-branch head (left) and $Q$-branch head (right) of the [19.22]1- $X^{1} \Sigma^{+}(0,0)$ band. Also show is the associated vibronic levels.

\section{Figure 4.}

The DF spectra resulting from excitations near the $R$-branch head (left) and the $Q$-branch head $[19.40] 1-X^{1} \Sigma^{+}(0,0)$ band. Also show is the associated vibronic levels.

\section{Figure 5.}

Observed and calculated radiative decay curves resulting from the laser excitation near the $R$ branch heads of the $[17.58] 0^{+}-X^{1} \Sigma^{+}(0,0)$ and $[18.42] 0^{+}-X^{1} \Sigma^{+}(0,0)$ bands and the $Q$-branch heads [19.22] $1-X^{1} \Sigma^{+}(0,0)$ and $[19.40] 1-X^{1} \Sigma^{+}(0,0)$ bands. In each case resonant fluorescence was monitored. 


\section{References}

1. J. Engel, M. J. Ramsey-Musolf and U. van Kolck, Prog. Part. Nucl. Phys. 71, 21-74 (2013).

2. B. M. Roberts, V. A. Dzuba and V. V. Flambaum, Annu. Rev. Nucl. Part. Sci. 65, 63-86 (2015).

3. J. S. M. Ginges and V. V. Flambaum, Phys. Rep. 397 (2), 63-154 (2004).

4. M. G. Kozlov and L. N. Labzowsky, J. Phys. B: At., Mol. Opt. Phys. 28 (10), 1933-1961 (1995).

5. J. Baron, W. C. Campbell, D. DeMille, J. M. Doyle, G. Gabrielse, Y. V. Gurevich, P. W. Hess, N. R. Hutzler, E. Kirilov, I. Kozyryev, B. R. O'Leary, C. D. Panda, M. F. Parsons, E. S. Petrik, B. Spaun, A. C. Vutha and A. D. West, Science (Washington, DC, U. S.) 343 (6168), 269-272 (2014).

6. A. E. Leanhardt, J. L. Bohn, H. Loh, P. Maletinsky, E. R. Meyer, L. C. Sinclair, R. P. Stutz and E. A. Cornell, J. Mol. Spectrosc. 270 (1), 1-25 (2011).

7. V. F. Dmitriev, I. B. Khriplovich and V. B. Telitsin, Phys. Rev. C: Nucl. Phys. 50 (5), 2358-2361 (1994).

8. V. V. Flambaum, D. DeMille and M. G. Kozlov, Phys Rev Lett 113 (10), 103003 (2014).

9. L. V. Skripnikov, A. N. Petrov, A. V. Titov and V. V. Flambaum, Phys. Rev. Lett. 113 (26), 263006/263001-263006/263005, 263005 pp. (2014).

10. V. V. Flambaum, Phys. Lett. B 320 (3-4), 211-215 (1994).

11. L. V. Skripnikov, A. N. Petrov, N. S. Mosyagin, A. V. Titov and V. V. Flambaum, Phys. Rev. A: At., Mol., Opt. Phys. 92 (1-A), 012521/012521-012521/012526 (2015). 
12. T. Fleig, M. K. Nayak and M. G. Kozlov, Physical Review A 93 (1), 012505 (2016).

13. J. K. Bates and D. M. Gruen, J. Chem. Phys. 70 (9), 4428-4429 (1979).

14. M. Zhou and L. Andrews, J. Phys. Chem. A 102 (45), 9061-9071 (1998).

15. R. S. Ram, J. Lievin and P. F. Bernath, J. Mol. Spectrosc. 215 (2), 275-284 (2002).

16. E. S. Shuman, J. F. Barry and D. DeMille, Nature (London, U. K.) 467 (7317), 820-823 (2010).

17. D. L. Kokkin, T. C. Steimle and D. DeMille, Phys. Rev. A: At., Mol., Opt. Phys. 90 (6A), 062503/062501-062503/062510 (2014). 
Figure 1
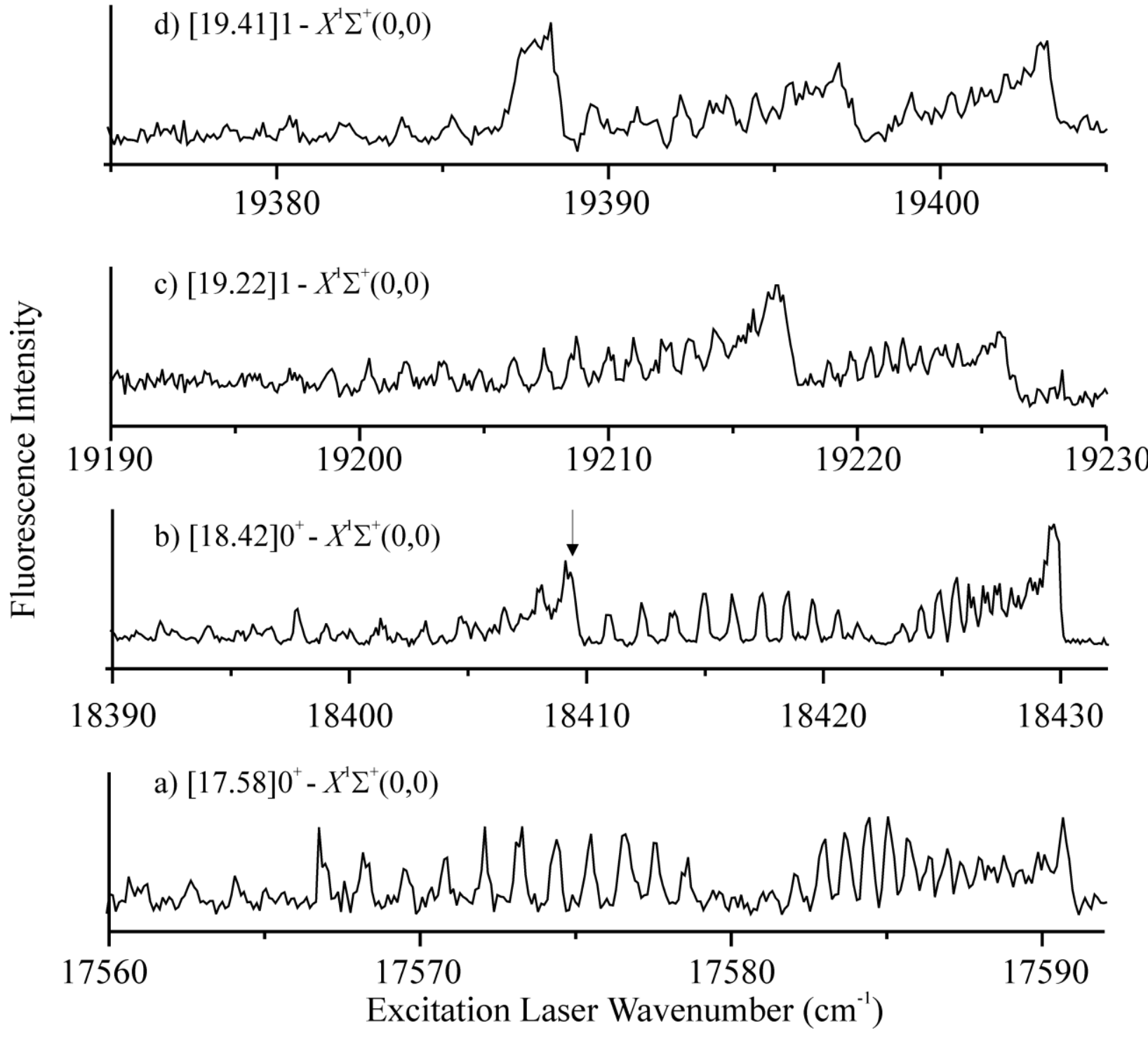
Figure 2

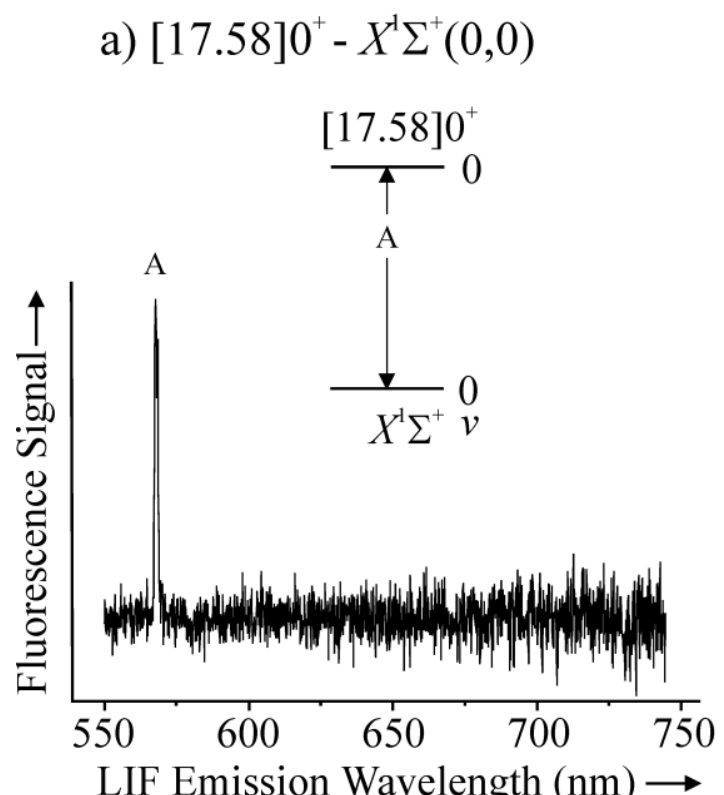

b) $[18.42] 0^{+}-X^{\prime} \Sigma^{+}(0,0)$

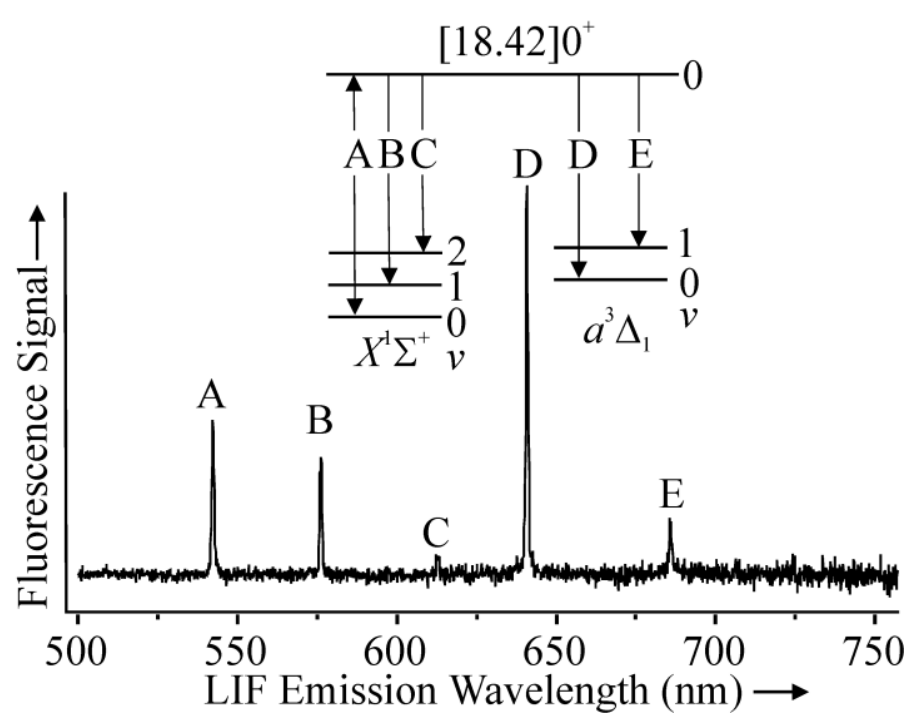


Figure 3
a) $[19.22] 1-X^{\top} \Sigma^{+}(0,0) R$-branch head
b) $[19.22] 1-X^{1} \Sigma^{+}(0,0) Q$-branch head
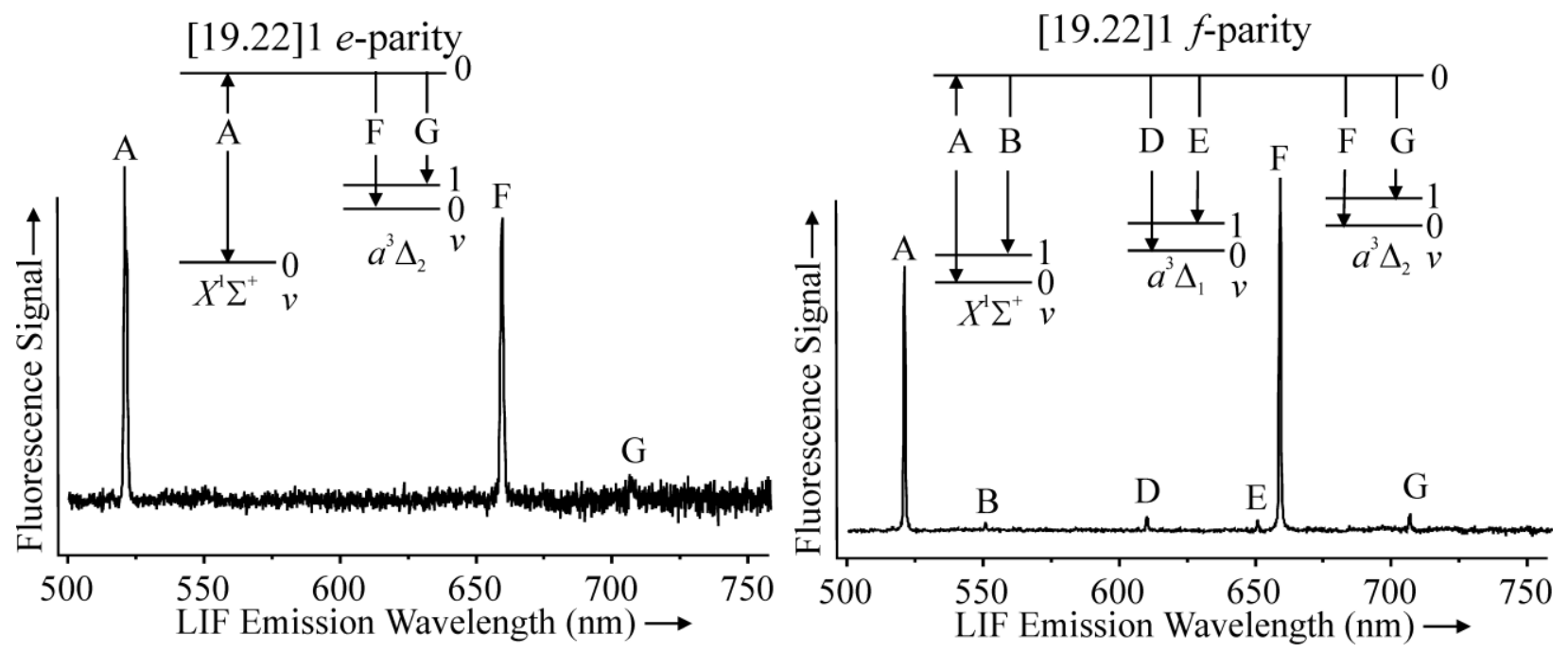
Figure 4
a) $[19.40] 1-X^{\prime} \Sigma^{+}(0,0) R$-branch head
b) $[19.40] 1-X^{\prime} \Sigma^{+}(0,0) Q$-branch head
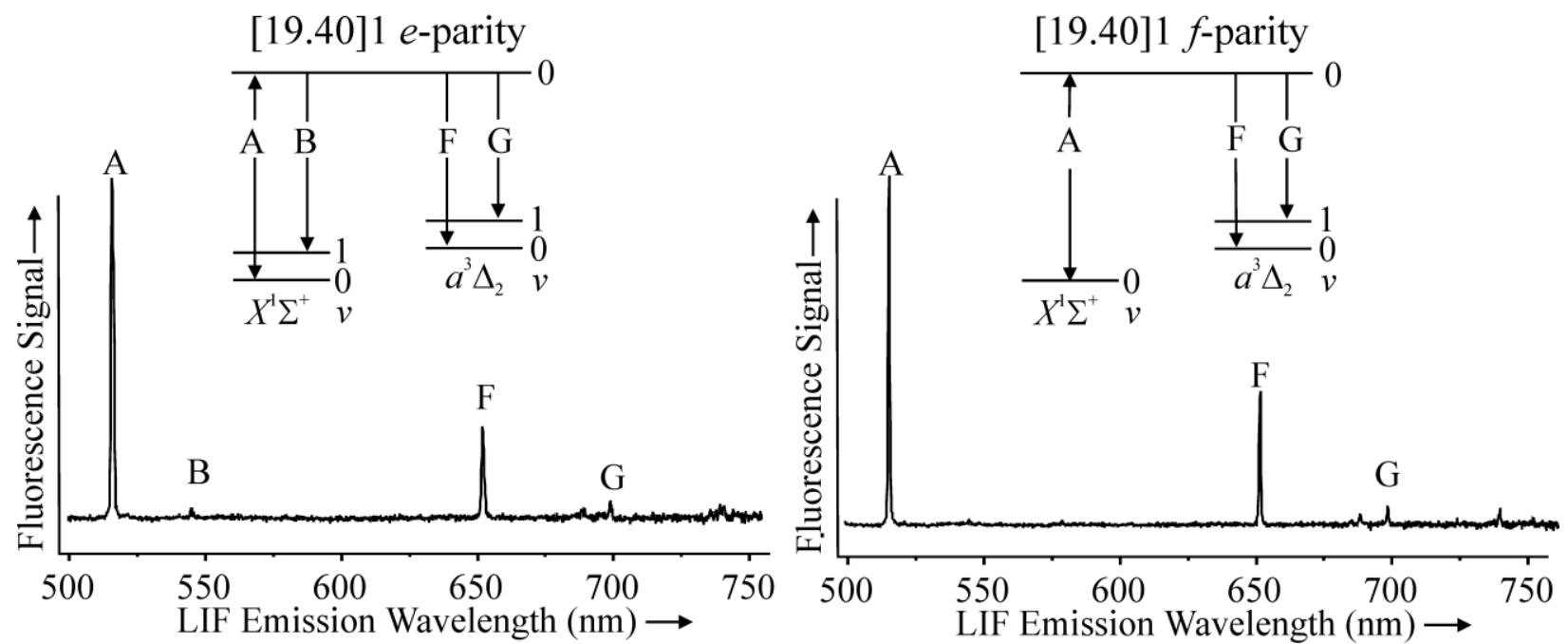
Figure 5
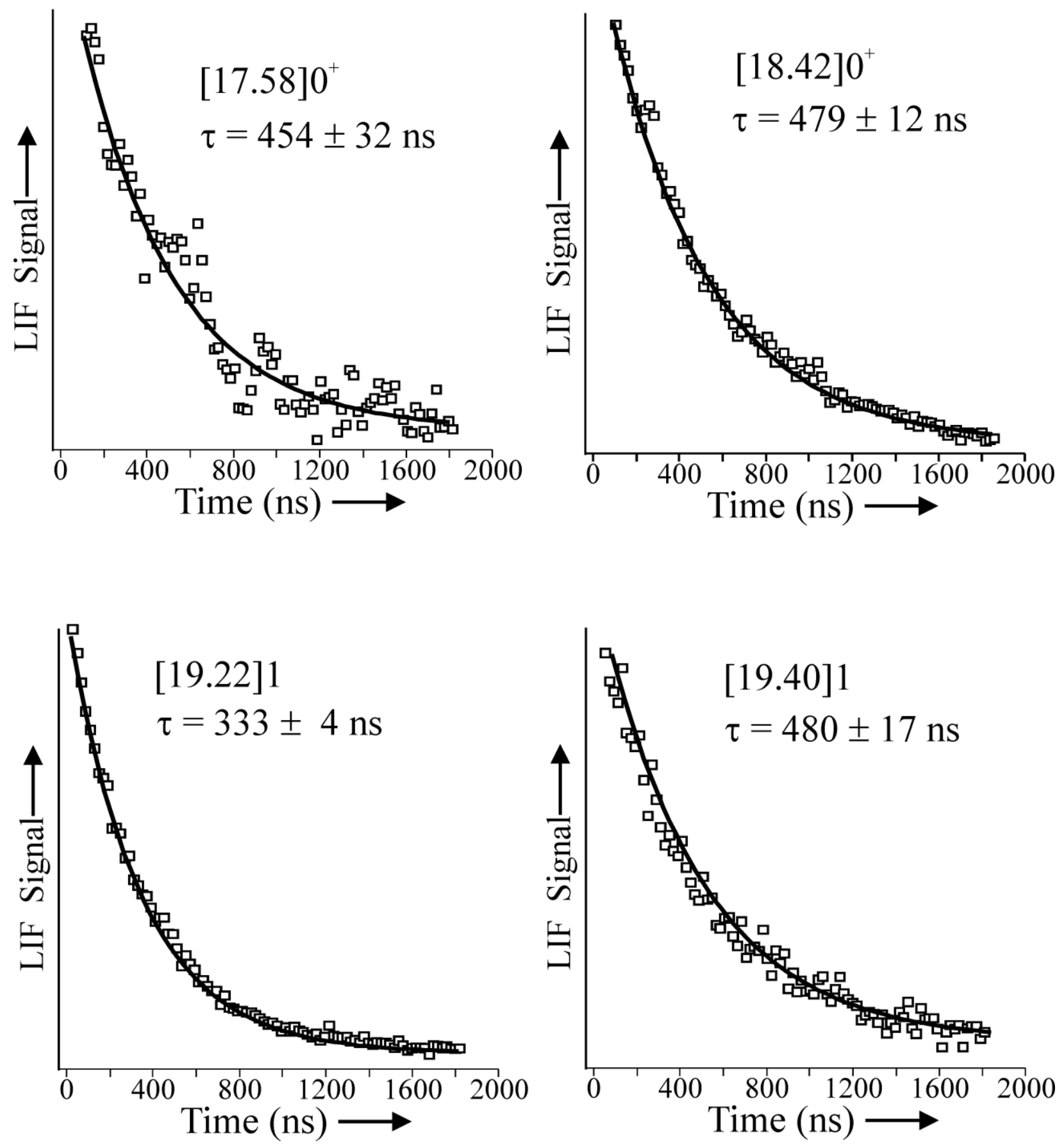


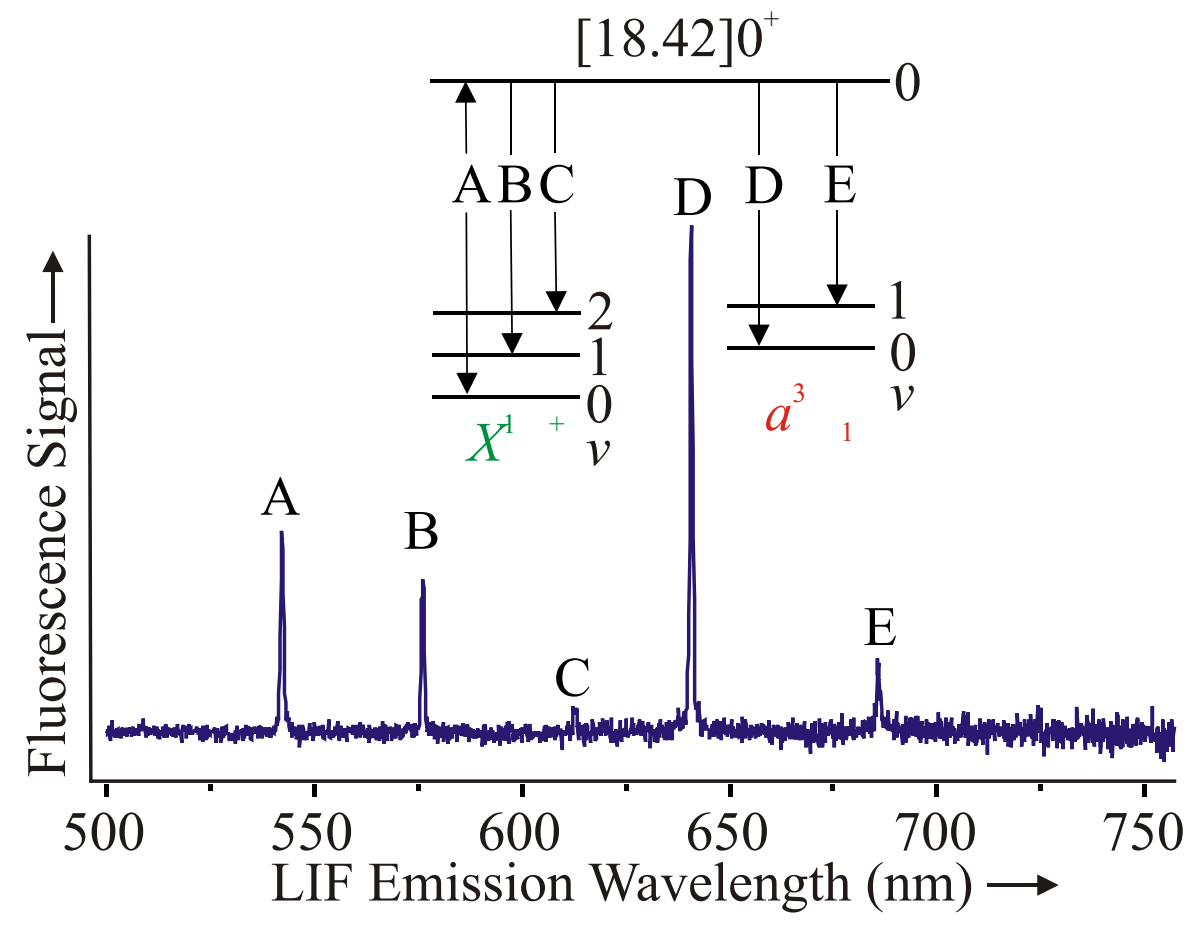

